\title{
Visual-Induced Excitation Leads to Firing Pauses in Striatal Cholinergic Interneurons
}

\author{
Jan M. Schulz, Manfred J. Oswald, and John N. J. Reynolds \\ Department of Anatomy and Structural Biology, Brain Health Research Centre, University of Otago, Dunedin 9054, New Zealand
}

Tonically active neurons in the primate striatum, believed to be cholinergic interneurons (CINs), respond to sensory stimuli with a pronounced pause in firing. Although inhibitory and neuromodulatory mechanisms have been implicated, it is not known how sensory stimuli induce firing pauses in CINs in vivo. Here, we used intracellular recordings in anesthetized rats to investigate the effectiveness of a visual stimulus at modulating spike activity in CINs. Initially, no neuron was visually responsive. However, following pharmacological activation of tecto-thalamic pathways, the firing pattern of most CINs was significantly modulated by a light flashed into the contralateral eye. Typically, this induced an excitation followed by a pause in spike firing, via an underlying depolarization-hyperpolarization membrane sequence. Stimulation of thalamic afferents in vitro evoked similar responses that were independent of synaptic inhibition. Thus, visual stimulation likely induces an initial depolarization via a subcortical tecto-thalamo-striatal pathway, pausing CIN firing through an intrinsic afterhyperpolarization.

\section{Introduction}

The basal ganglia are a group of subcortical nuclei that play an essential role in forebrain function, through the selection of motor programs (Grillner et al., 2005). The striatum, the major input nucleus of the basal ganglia, consists mainly of spiny projection neurons (SPNs) (97\% of neurons) and small groups of local interneurons (Oorschot, 1996). Unique to the striatum are the cholinergic interneurons (CINs), which release the neuromodulator acetylcholine $(\mathrm{ACh})$. These interneurons powerfully modulate synaptic integration in SPNs (Pakhotin and Bracci, 2007; Shen et al., 2007) and are implicated in the pathogenesis of Parkinson's disease (Pisani et al., 2007). Hence, determining the mechanisms that regulate CIN firing in vivo is essential to our understanding of striatal functions in action selection and neurological disorders.

Tonically active neurons (TANs), likely to be CINs, exhibit prominent sensory responses in monkeys engaged in learning an association between a stimulus and a reward (Kimura, 1990; Aosaki et al., 1994; Morris et al., 2004; Joshua et al., 2008). These responses involve a "pause" in their tonic firing that is often, but not always, flanked by an initial and a rebound excitation. Several mechanisms have been suggested to account for this spike suppression. First, the observation of coincident excitatory responses from midbrain dopamine (DA) neurons in behaving primates (Morris et al., 2004) suggests that phasic DA could directly sup-

Received Feb. 7, 2011; revised April 11, 2011; accepted June 15, 2011.

Author contributions: J.M.S. and J.N.J.R. designed research; J.M.S. and M.J.0. performed research; J.M.S. and M.J.O. analyzed data; J.M.S. and J.N.J.R. wrote the paper.

This work was supported by The Marsden Fund of the Royal Society of New Zealand (J.N.J.R.) and the Neurological Foundation of New Zealand (M.J.O., J.N.J.R.). J.M.S. received a University of Otago Postgraduate Scholarship and Publishing Bursary. We thank Dr. B. Hyland and Dr. K.-Y. Tseng for comments on early versions of this manuscript.

Correspondence should be addressed to Dr. John N. J. Reynolds, Department of Anatomy and Structural Biology, Brain Health Research Centre, University of Otago, P. O. Box 913, Dunedin 9054, New Zealand. E-mail: john.reynolds@stonebow.otago.ac.nz.

DOI:10.1523/JNEUROSCI.0661-11.2011

Copyright $\odot 2011$ the authors $\quad 0270-6474 / 11 / 3111133-11 \$ 15.00 / 0$ press spiking in TANs. In support, DA directly regulates multiple intrinsic conductances in the CIN via $\mathrm{D}_{1}$ - and $\mathrm{D}_{2}$-type $\mathrm{DA}$ receptor activation (Yan et al., 1997; Aosaki et al., 1998; Maurice et al., 2004; Deng et al., 2007) and appears to suppress local ACh release in the striatum (Kudernatsch and Sutor, 1994; DeBoer and Abercrombie, 1996). However, the effect of DA on CINs is depolarizing and causes sustained spike rate increases (Aosaki et al., 1998; Deng et al., 2007); therefore, phasic dopamine is unlikely to drive the pause directly. Second, CINs have recently been shown to inhibit their own activity by engaging an as-yet-unidentified population of GABAergic neurons (Sullivan et al., 2008). Strong intrastriatal stimulation was required to induce synchronized CIN firing and elicit this effect; how this mechanism is engaged in vivo and acquired through learning is yet to be determined. Finally, we have reported that membrane depolarization of CINs induced by glutamatergic inputs can trigger an intrinsic afterhyperpolarization (AHP) that suppresses spike firing for $>200 \mathrm{~ms}$ (Reynolds et al., 2004; Oswald et al., 2009).

Despite the number of mechanisms capable of inducing pauses in CINs, it remains undemonstrated how any of these mechanisms may be engaged by sensory inputs. Here, we show that visual activation of subcortical tecto-thalamic pathways after pharmacological disinhibition of the superior colliculus (SC) powerfully modulates the spiking behavior and underlying membrane potential of intracellularly recorded CINs in rats. This glutamatergic pathway could therefore elicit the pause response observed in TANs.

Some of the present results have previously appeared in abstract form (Reynolds et al., 2009).

\section{Materials and Methods}

Experimental preparation for in vivo recordings. Every effort was made to minimize the pain and discomfort of the experimental animals used in this study, in accordance with approvals granted by the University of Otago Animal Ethics Committee. Male Long-Evans rats (230-410 g) 
were anesthetized with urethane (1.4-1.9 g/kg, i.p.; Biolab), supplemented with additional urethane $(0.2 \mathrm{~g} / \mathrm{kg}) 1$ - to 2-hourly, as required. The core temperature was maintained at $36^{\circ} \mathrm{C}$ by a homoeothermic blanket. All wounds and pressure points were infiltrated with a long-acting local anesthetic (bupivacaine, $0.5 \%$ ). The head was fixed in a stereotaxic frame, and surgery was performed as previously described (Schulz et al., 2009). Briefly, a hole was drilled into the skull above the left visual cortex at $8.0 \mathrm{~mm}$ posterior bregma (AP, -8.0$)$ and $4.2 \mathrm{~mm}$ mediolateral from the midline $(\mathrm{ML},+4.2)$ and a silver wire EEG electrode in contact with the dura was secured with dental cement. Another hole was drilled above the left SC, around AP -6.5 and ML + 1.4, to accept a pipette pulled from precalibrated glass capillaries (volume, $5 \mu \mathrm{l}$; diameter, $1.0 \mathrm{~mm}$; Modulohm) and filled with bicuculline (BIC) (0.01\% in saline; Sigma-Aldrich). The BIC ejection pipette was lowered $4.1 \mathrm{~mm}$ from brain surface into the SC deep layers and secured in place with dental cement. To document the restoration of visual sensitivity enabled by BIC (Schulz et al., 2009), the local field potential was routinely recorded in the SC deep layers through an attached recording electrode.

Intracellular recordings. Intracellular records were made using micropipettes pulled from 3.0-mm-diameter glass (Harvard Apparatus) and filled with $1 \mathrm{~m} \mathrm{~K}$-acetate (45-95 $\mathrm{M} \Omega$ resistance), in some cases containing $2 \%$ biocytin. The micropipette was advanced through the striatum from initial penetrations between $\mathrm{AP}+0.2$ to +1.0 and $\mathrm{ML}+2.7$ to +3.9 , until a stable recording was obtained from a neuron. Recordings from CINs were obtained serendipitously while searching for spiny projection neurons for other purposes (Schulz et al., 2009). All electrophysiologically identified CINs exhibited a prominent sag in the hyperpolarizing membrane potential response to negative current injections representing the contribution of $I_{\mathrm{H}}$ (range, $3.0-12.0 \mathrm{mV}$ ), and an AHP after depolarizing current injections (range, 3.2-13.9 mV) (see Fig. 1) (Kawaguchi et al., 1995; Reynolds and Wickens, 2004; Goldberg and Wilson, 2005). Current-voltage relationships were obtained by injecting hyperpolarizing and depolarizing current pulses through the micropipette, using an Axoclamp-2B amplifier (Molecular Devices) configured in current-clamp mode. To minimize the influence of $I_{\mathrm{H}}$ or spontaneous spikes, input resistance was determined from the slope of a regression line fitted to four minimal membrane potentials produced by a series of negative current pulses $(-0.6,-0.4,-0.2,0 \mathrm{nA})$.

Light stimulation. Light stimuli $(10 \mathrm{~ms}$ duration; $0.1-0.2 \mathrm{~Hz})$ were delivered from a white LED $(1500 \mathrm{mcd})$ positioned $<3 \mathrm{~cm}$ directly in front of the animal's right eye. All waveform data were digitized at $10 \mathrm{kHz}$ by a Digidata 1322A (Molecular Devices), displayed using pClamp 10 software (Molecular Devices), and stored to disk. Following baseline recording ( $\geq 24$ responses at $0.1-0.2 \mathrm{~Hz}, 5-15 \mathrm{~min}$ ), BIC (200-300 nl) was ejected into the SC at a rate of $\sim 200 \mathrm{nl} / \mathrm{min}$. The number of light trials that could be collected after BIC was often restricted to $<40$ trials (3-60 min stable recording after BIC; $n=8$ experiments) possibly due to transient mechanical instability during the ejection process.

Histology. In all experiments, tips of BIC ejection pipettes were verified to be within the intermediate/deep layers of the SC using light microscopy of unstained sections or sections stained with cresyl violet $(0.1 \%)$. During recordings of at least $30 \mathrm{~min}$, neurons were passively filled with biocytin. Vibratome sections $(50 \mu \mathrm{m})$ containing biocytinfilled neurons were processed using standard histological procedures (Horikawa and Armstrong, 1988) and labeled cells were identified by fluorescent microscopy.

In vitro patch-clamp recordings. Acute brain slices were prepared from P14-P24 male Wistar rats. Brains were perfused transcardially with ice-cold dissection solution (in mм: 225 sucrose, 10 glucose, $2.5 \mathrm{KCl}, 7$ $\mathrm{MgCl}_{2}, 0.5 \mathrm{CaCl}_{2}, 28 \mathrm{NaHCO}_{3}, 1 \mathrm{NaH}_{2} \mathrm{PO}_{4}$; bubbled with $95 \% \mathrm{O}_{2}$ and $5 \% \mathrm{CO}_{2}$ ). Slices $(300-400 \mu \mathrm{m})$ were cut using a vibratome (VT1000S; Leica) at an oblique angle of $\sim 20^{\circ}$ to the horizontal plane, to maximally preserve thalamo-striatal connections (Smeal et al., 2007; Ding et al., 2008). Slices were transferred to artificial CSF (ACSF) (containing in mM: $125 \mathrm{NaCl}, 2 \mathrm{MgSO}_{4}, 2 \mathrm{CaCl}_{2}, 2.5 \mathrm{KCl}, 10$ glucose, $26 \mathrm{NaHCO}_{3}, 1$ $\mathrm{NaH}_{2} \mathrm{PO}_{4}$; bubbled with $95 \% \mathrm{O}_{2}$ and $5 \% \mathrm{CO}_{2}$ ) and incubated for $45 \mathrm{~min}$ at $35^{\circ} \mathrm{C}$. Slices were then maintained at room temperature for a minimum recovery period of $1 \mathrm{~h}$. For recording, slices were transferred to a temperature controlled recording chamber (TC-2; Bioscience Tools)

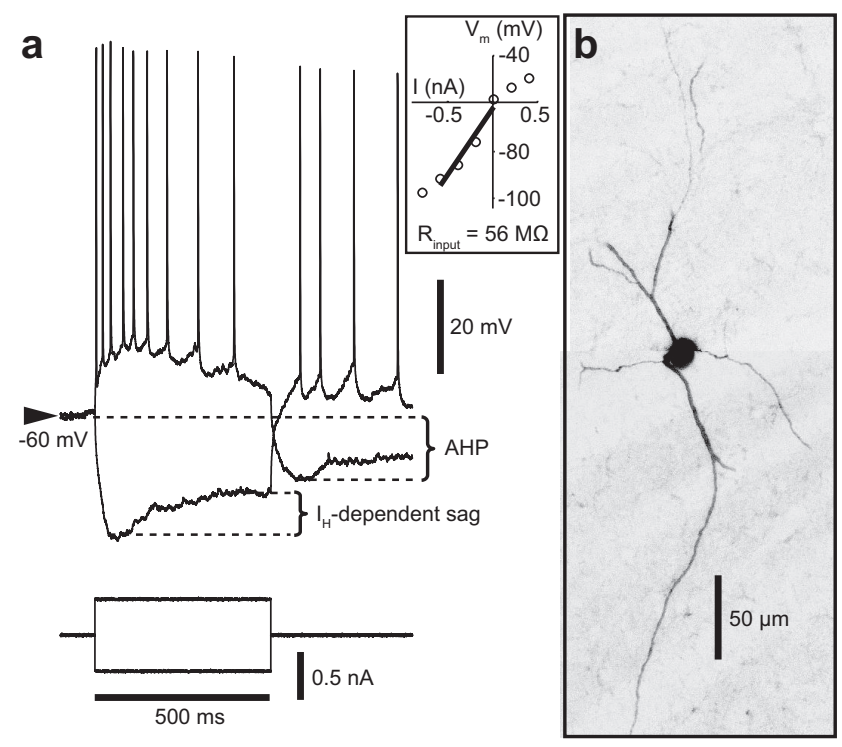

Figure 1. Electrophysiological properties of CINs. $\boldsymbol{a}$, Membrane potential response to a pos itive and a negative current step in a CIN. Note the characteristic sag in the hyperpolarizing membrane potential response that depends on the hyperpolarization and cyclic nucleotideactivated cation current $\left(I_{H}\right)$, and the AHP response after a depolarizing current injection. The inset shows the current-voltage relationship; the input resistance was derived from the slope of the regression line. $\boldsymbol{b}$, Merged Z-projections from confocal image stacks of the same neuron. The fusiform soma is oriented along the Z-plane and appears round.

perfused with oxygenated $\operatorname{ACSF}\left(2 \mathrm{ml} / \mathrm{min} ; 33^{\circ} \mathrm{C}\right)$. Striatal CINs $(n=31$; spontaneously spiking, 16; silent, 15) were visualized using infrareddifferential interference contrast optics (BX51WI; Olympus Optical) and an IR-1000 CCD camera (Dage-MTI). Recording pipettes had a resistance of 6-9 $\mathrm{M} \Omega$ when filled with a solution containing the following (in $\mathrm{mM}$ ): $130 \mathrm{~K}$-methylsulfate, $12 \mathrm{KCl}, 2 \mathrm{Na}_{2} \mathrm{ATP}, 0.4 \mathrm{Na}_{2} \mathrm{GTP}, 2 \mathrm{MgCl}_{2}$, and 10 HEPES, pH 7.4, 290-300 mOsm/L. Current-clamp recordings were made in the whole-cell configuration using a MultiClamp 700B amplifier (Molecular Devices), with series resistance compensation of 15-25 M $\Omega$. The signals were low-pass filtered at $4 \mathrm{kHz}$ and digitized at $10 \mathrm{kHz}(1322 \mathrm{~A}$ Digidata and pClamp 9 acquisition software; Molecular Devices). Thalamic afferents were stimulated through a stimulation electrode place close to the reticular thalamic nucleus (Ding et al., 2008, 2010). All drugs were obtained from Tocris Bioscience.

Data analysis. Data were analyzed off-line using MATLAB 7.1 with Signal Processing 6.4 and Statistics 5.1 Toolboxes. Axon binary files were imported into MATLAB using a function obtainable from http://www. mathworks.de/matlabcentral/fileexchange/.

Assessment of membrane potential fluctuations. The membrane potential of all neurons at each time point was corrected off-line by the estimated tip potential present at the time of recording. Spikes were detected using a threshold criterion after high-pass filtering (third-order Butterworth; cutoff, $100 \mathrm{~Hz}$ ). The time of each spike maximum was saved, and the spike was removed from the unfiltered "membrane potential fluctuations" at the spike threshold level (instantaneous slope of $>8 \mathrm{mV} / \mathrm{ms}$ ). Some neurons exhibited extended periods without spikes in vivo $(n=2)$ and in vitro $(n=15)$. Subthreshold membrane potential fluctuations from these neurons were analyzed separately from light trials with spontaneous spikes.

Membrane potential fluctuations were low-pass filtered (fourth-order Butterworth; $45 \mathrm{~Hz}$ cutoff) and downsampled to $500 \mathrm{~Hz}$. Parameters of visual-evoked membrane potential responses were estimated on the mean membrane potential fluctuations corrected by the mean prelight membrane potential offset. The "postsynaptic potential (PSP) amplitude" was defined as the maximum of the mean membrane potential during the first $500 \mathrm{~ms}$ following the light flash. To reduce the impact of noise around the maximum, the "latency" was measured to the PSP half-amplitude. The "AHP latency" was defined as the first time 
$a_{1}$

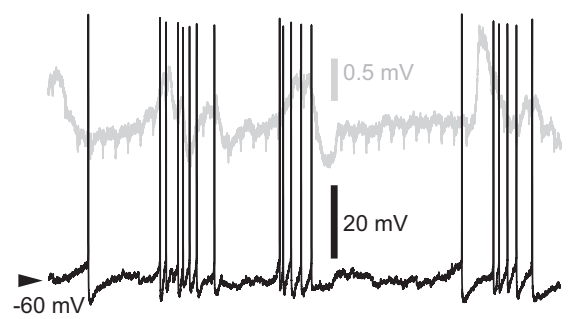

$\mathbf{a}_{2}$

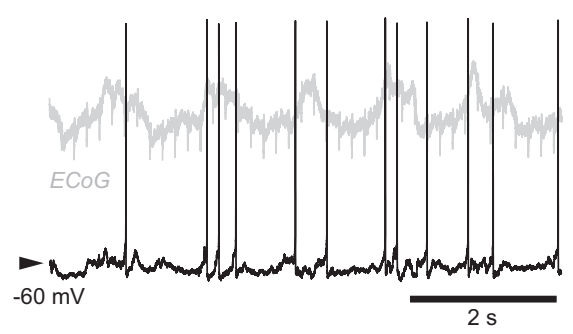

08n30n1

$a_{3}$

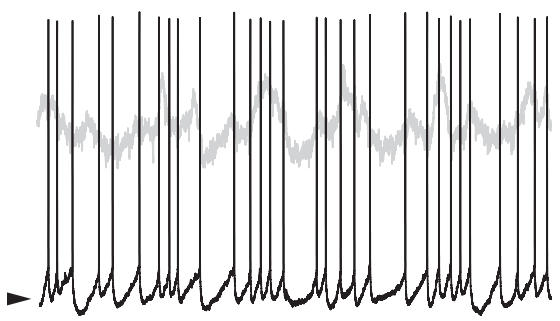

$b_{1}$

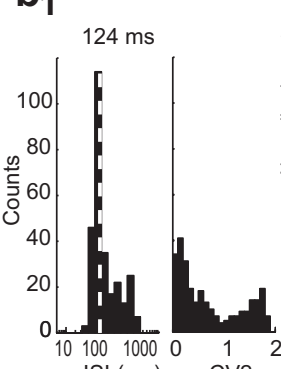

ISI (ms)

$b_{2}$

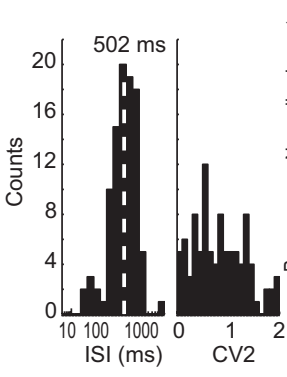

$b_{3}$

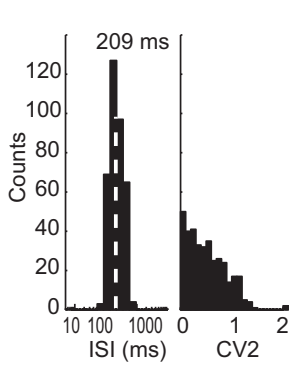

$\mathbf{C}_{1}$
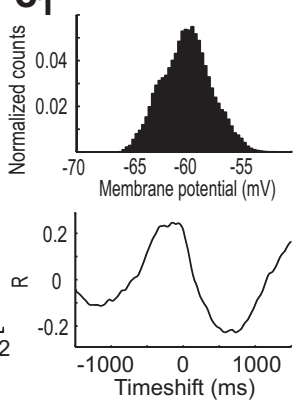

$\mathrm{C}_{2}$
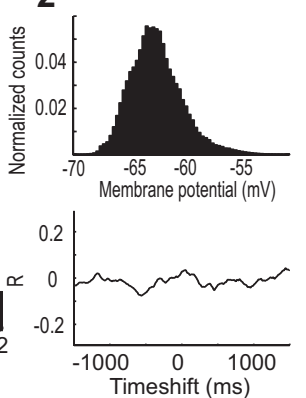

$\mathrm{C}_{3}$
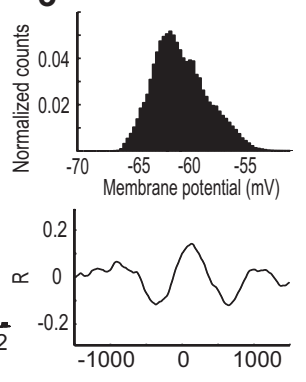

Figure 2. Relationship between spontaneous spiking in CINs and slow-wave ECOG activity. $\boldsymbol{a} 1-\boldsymbol{a}$ 3, Seven-second-long segments of intracellular and simultaneous EC $0 \mathrm{G}$ activity from a continuous recording of $90 \mathrm{~s}$ for three CINs (numbered $1-3$ ). Note the different activity patterns across neurons, ranging from phasic (top) and irregular (middle) to regular spiking (bottom). $\boldsymbol{b} 1-\boldsymbol{b} \mathbf{3}$, Distributions of ISIs and CV2 values for the whole 90 s of the same recordings as in $\boldsymbol{a 1 - a 3}$. The median ISI is indicated (dashed line). c1-c3, Membrane potential distribution (top) and cross-correlograms between ECOG and subthreshold membrane potential fluctuations (bottom) for the same three CINs.

the membrane potential crossed zero immediately before reaching $(-1.5)^{\star} \mathrm{SD}$ of the mean membrane potential fluctuations following the light. The latency to the "AHP end" was defined as the first time the membrane potential crossed zero immediately before reaching $(+1)^{\star} \mathrm{SD}$ of the mean membrane potential fluctuations after the AHP onset. The "AHP duration" was defined as the difference between AHP end and AHP latency. The "PSP area" was calculated as the sum of all positive values from $80 \mathrm{~ms}$ after the light flash to the AHP start, and the "AHP area" was the absolute value of the sum of all negative values from the AHP start to end. PSP and AHP areas of traces obtained in vitro were derived as previously described (Oswald et al., 2009).

Assessment of spiking activity. The effect of visual stimulation on spike data was examined by producing peristimulus time histograms (PSTHs) (20 ms bin width). The PSTHs of individual experiments were smoothed (sliding Hamming window of 5 bins) and transformed into percentage change of the overall mean prestimulus spike rate. Latencies, durations, and magnitudes of light-induced spike rate changes were estimated for individual experiments with significant coefficient of variation 2 (CV2) modulation (see below) by cursor measurements analog to the membrane potential measurements. To detect effects of the stimulus on spiking regularity, a time-resolved transform of the CV2 of the interspike intervals (ISIs) was produced (Shin et al., 2007). The CV2 ranges between
0 and 2 (with 2 indicating maximum irregularity), and is an estimate of the local spike irregularity based on three adjacent spikes: $\mathrm{CV} 2_{n}$ $=2^{\star}$ absolute $\left(\operatorname{ISI}_{n}-\operatorname{ISI}_{n-1}\right) /\left(\operatorname{ISI}_{n}+\operatorname{ISI}_{n-1}\right)$, where ISI $_{n}$ is the interval between spikes 2 and 3 and ISI $_{n-1}$ is the interval between spikes 1 and 2 in a series of three spikes. Each CV2 ${ }_{n}$ was assigned to all time bins between spikes 2 and 3 (i.e., $\mathrm{ISI}_{n}$ ). We extended the analysis used by Shin et al. (2007) on spontaneous activity, by aligning all trials to stimulus onset and averaging across trials after downsampling to $20 \mathrm{~ms}$ resolution. The time-resolved CV2 is in principle similar to another method that calculates a continuous signal from the ratio of adjacent ISIs (Davies et al., 2006).

Spontaneous activity parameters. For each light trial, the mean prelight spike rate, CV2, and membrane potential were calculated as the mean over the $1500 \mathrm{~ms}$ preceding the light flash. These prelight means were considered to be unaffected by any previous light stimulation (>3.5 $\mathrm{s}$ after light).

Spike-electrocorticogram coherence analysis. For each spike of a 30-90 s recording of spontaneous activity without stimulation, the surrounding electrocorticogram (ECoG) signal $( \pm 1 \mathrm{~s})$ was obtained. The spike-triggered average (STA) was calculated as the mean of this ECoG segment over all spikes. As a control, the procedure was repeated 100 times on the same spontaneous ECoG recording using surrogate spike trains. Surrogate spike trains were obtained by randomization of the order of ISIs from the original spike train. Only peaks of STAs that exceeded all control STAs were considered significant. For the coherence analysis, we took advantage of the fact that oscillatory components of the ECoG will disappear in the STA unless the spike is phase-locked to the same oscillation. To obtain the spike-ECoG coherence, the power spectrum of the STA was calculated using fast-Fourier transformation and normalized by the mean power spectrum of all included ECoG segments (Fries et al., 2001). Values for individual frequencies in the spike-ECoG coherence range from 0 , indicating no systematic phase relationship between spikes and ECoG, to 1, indicating perfect phase-locking. A detailed description of this method can be found in the study by Fries et al. (2001). The maximum peak of the spike-ECoG coherence signal was measured for frequencies $<3 \mathrm{~Hz}$ to assess the relationship of CIN spiking to the dominant synchronous slow-wave ECoG activity during urethane anesthesia.

Statistical analysis. To test for systematic changes in spontaneous activity parameters induced by BIC ejection, prelight means measured before and after BIC were compared using the Wilcoxon rank sum test for equal medians. The probability level for statistical significance was set at $p=0.05$.

To test for stimulus-induced changes in individual experiments, single-trial membrane potential fluctuations and time-resolved CV2 (both: 20 ms resolution) were corrected by the prestimulus medians, and values of each bin were tested for significant deviation from zero using the Wilcoxon signed rank test for paired samples. The significance level was set at $p=0.001$ in all tests to restrict the number of false-positives during repetitive testing. The overall significance level was $p=0.1$, as all 100 bins during the first $2 \mathrm{~s}$ after the stimulus were tested for a significant effect of the stimulation. 
For group analysis, the normalized PSTH, mean membrane potential, and mean timeresolved CV2 were corrected by the prestimulus mean. The grand means of individual signals were considered to be significantly affected if the signal exceeded 2 SDs from the prestimulus baseline. A mixed model was used to analyze natural log-transformed means of PSP and AHP areas for treatment effects in vitro with random effects assigned to individual neurons.

Regression models. The data of potentially correlated parameters were fitted by a linear least-square fit to test for a significant interaction. Visual inspection of the scatter plot suggested that the relationship between spike-ECoG peak coherence and mean CV2 followed an exponential function. To test for a significant interaction, spike-ECoG peak coherence values were log-transformed before linear regression analysis. In all other cases, no indications for a nonlinear relationship between parameters were apparent from visual inspection of scatter plots.

\section{Results}

In vivo intracellular recordings were obtained from 11 CINs encountered fortuitously over a period of 5 years. These were identified using established electrophysiological criteria (Kawaguchi et al., 1995; Reynolds and Wickens, 2004; Goldberg and Wilson, 2005), including a hyperpolarization and cyclic nucleotide-activated cation current $\left(I_{\mathrm{H}}\right)$-dependent sag response (Fig. 1) and broad spike waveforms (halfwidth, $1.1 \pm 0.3 \mathrm{~ms}$; mean $\pm \mathrm{SD} ; n=11)$ compared with SPNs $(0.8 \pm 0.1 \mathrm{~ms} ; n=68$; $p<0.0001$; Wilcoxon's rank sum test) recorded over the same time (Schulz et al., 2009, 2010). The identity of two CINs was confirmed by intracellular labeling with biocytin and histological verification (Fig. 1b). a
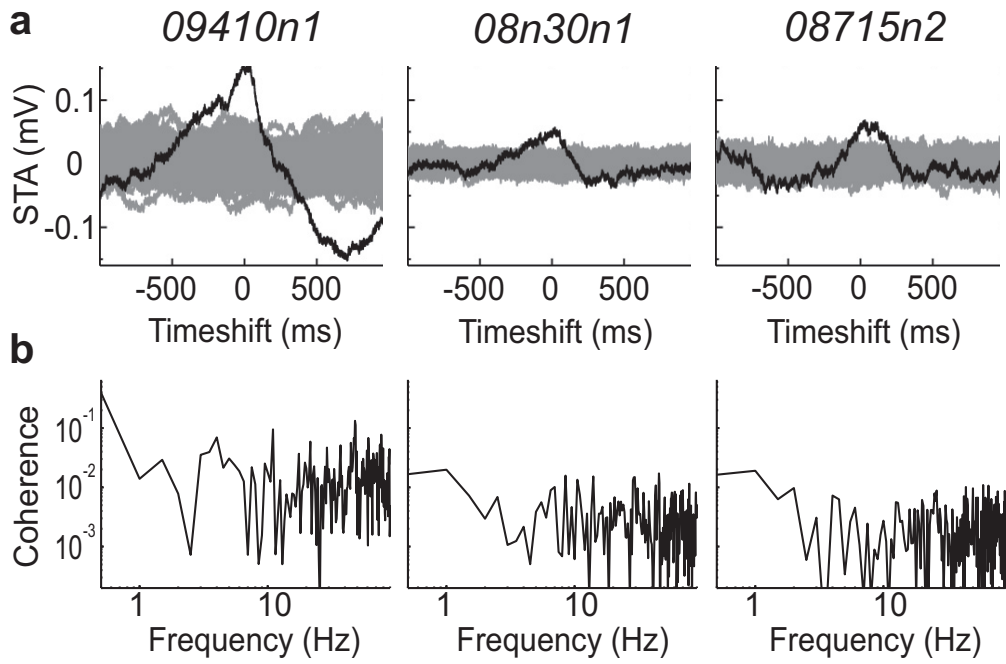

C

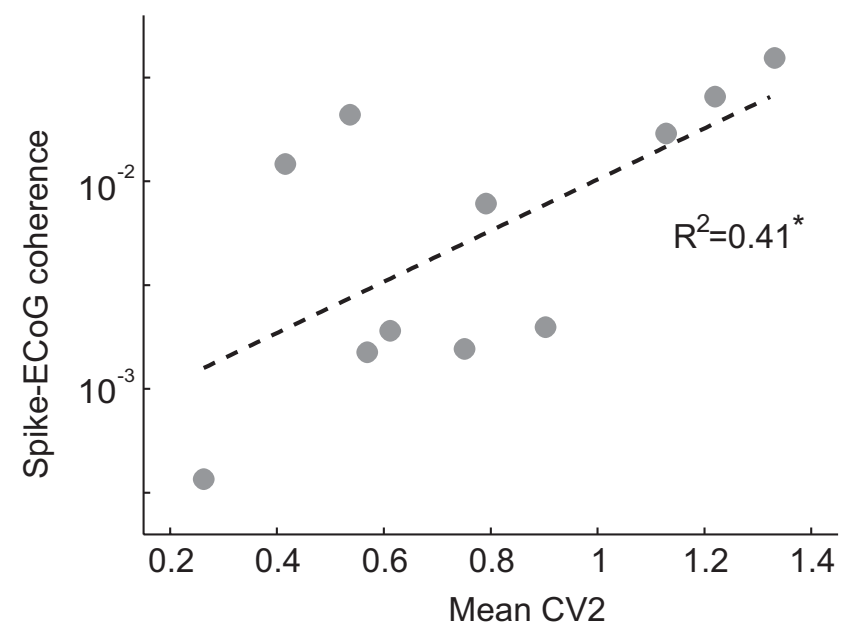

Figure 3. Coherence between spiking in CINs and ECOG correlates with spiking irregularity. $\boldsymbol{a}$, The STA of the ECOG (black) for the same neurons as in Figure 2. The STA had a peak around zero that exceeded chance level (gray traces), indicating that spike times were significantly correlated to ECOG activity in all three neurons. $\boldsymbol{b}$, Spike-ECoG coherence plotted on a double-logarithmic scale. c, The log-transformed peak value of the spike-ECoG coherence at frequencies $<3 \mathrm{~Hz}$ was significantly correlated to the mean $\mathrm{CV} 2$ value across neurons.

\section{In vivo activity pattern}

Nine CINs were tonically active throughout the recording, in accordance with their well characterized pacemaker properties (Bennett et al., 2000; Goldberg and Wilson, 2005; Wilson, 2005; Wilson and Goldberg, 2006). Two additional CINs instead exhibited extended periods without spike discharge. The overall average firing rate ranged from 0.1 to $10.9 \mathrm{~Hz}(n=11)$. Spiking patterns covered a broad spectrum ranging from regular tonic spiking to phasic activity (Fig. 2). To quantitatively evaluate the spiking irregularity, we calculated the CV2 for every pair of adjacent ISIs. The CV2 is less susceptible to commonly observed changes in spontaneous spike rate in vivo than the coefficient of variation (Holt et al., 1996; Shin et al., 2007). The mean CV2 across neurons ranged from 0.26 to 1.71 , clearly demonstrating the diversity in firing patterns (Fig. $2 b$ ) despite very similar unimodal membrane potential distributions ( $n=10$ of 11) (Fig. $2 c$ ).

Such variability in firing patterns could be due to differences in intrinsic pacemaker mechanisms (Bennett et al., 2000; Goldberg and Wilson, 2005; Wilson, 2005; Wilson and Goldberg, 2006) or, in our in vivo preparation, more likely reflected the differential impact of background synaptic inputs (Wilson et al., 1990; Reynolds and Wickens, 2004) during sleep-like thalamo- cortical slow wave activity (Clement et al., 2008). We measured the influence of thalamo-cortical inputs twofold: (1) by calculating the STA of the ECoG from 30 to $90 \mathrm{~s}$ traces of spontaneous activity, and (2) by calculating the cross-correlation of the subthreshold membrane potential with the ECoG. In the majority of neurons ( $n=9$ of 11$)$, a positive peak in the STA indicated that spike times were significantly affected by thalamo-cortical slow wave activity (Fig. $3 a$ ). The spike-ECoG coherence (Fries et al., 2001) for the dominant low frequencies of the ECoG $(<3 \mathrm{~Hz})$ ranged from 0.004 to 0.39 across neurons (Fig. $3 b, c$ ), indicating that the degree of firing modulation varied greatly between neurons. A similar variability was observed in the absolute crosscorrelation between ECoG and the subthreshold membrane potential (0.002-0.57). Neurons with high spike-ECoG coherence also exhibited a stronger correlation between membrane potential and ECoG $\left(R^{2}=0.40 ; p<0.05\right)$ (compare Figs. $\left.2 c, 3 a\right)$. In addition, log-transformed spike-ECoG coherence was significantly correlated with the mean CV2 value across all experiments $\left(R^{2}=0.41 ; p=0.033\right.$; number of observations $\left.=11 ; F=6.29\right)$ (Fig. $3 c$ ). Together, these data clearly demonstrate that the more irregular firing CINs were significantly influenced by global ex- 

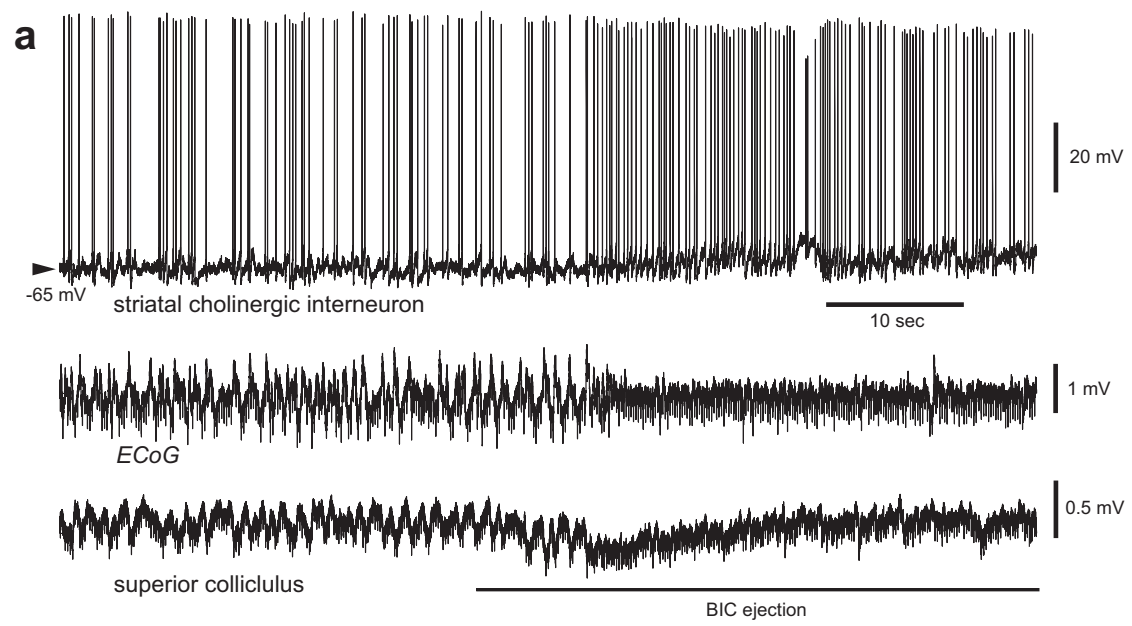

b

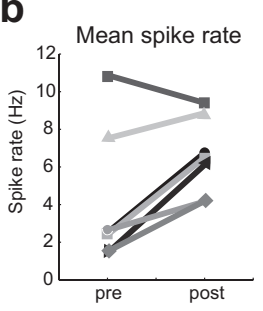

c

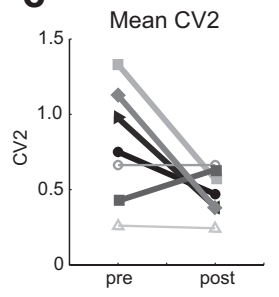

d

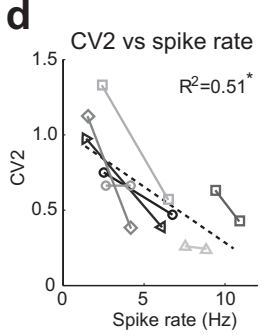

Figure 4. Effects of BIC ejection into the SC on spontaneous activity. a, Immediate effects on spontaneous activity in a (IN (top), cortex (middle), and SC (bottom) were already obvious during BIC ejection into the SC. $\boldsymbol{b}$, Across the whole recording, the mean spike rate was increased in six neurons and significantly decreased in the seventh neuron after BIC. $c$, The mean CV2 changed significantly in five neurons. Significant changes ( $p<0.05$, Wilcoxon's rank sum test) are indicated by filled circles and thick lines. Data from individual neurons have the same symbols and gray shade in all panels. $\boldsymbol{d}$, A scatter plot of mean CV2 versus mean spike rate. Before and after values from the same neuron are connected by a line. CV2 values were significantly correlated with mean spike rates (dashed line; $p=0.0042$ ). $e$, Overall minimal ISI before and after BIC. Note that the minimal ISI was never smaller than $10 \mathrm{~ms}$ (dashed line) despite spike rate increases after BIC.

citatory input, with phasic spiking activity occurring in phase with the slow ECoG waves, whereas neurons exhibiting tonic regular spiking were little affected by afferent network activity.

\section{Influence of brain state on spontaneous spike pattern}

We next investigated how the spike pattern was affected by the disruption of slow-wave activity during experimental-induced general activation. Local ejection of the $\mathrm{GABA}_{\mathrm{A}}$ antagonist BIC into the SC produced a marked reduction in spontaneous slowwave neural activity locally in the SC and in the ECoG (Fig. 4a) (Schulz et al., 2009). Under these conditions, the spontaneous spike rate was significantly increased in six of seven spontaneously spiking CINs $(p<0.001)$ (Fig. $4 b)$. One neuron remained silent throughout the post-BIC recording. Consistent with the idea that increased excitatory drive caused spike rate increases, the mean membrane potential was $2.4 \pm 1.1 \mathrm{mV}$ more depolarized after BIC in all six neurons that exhibited increased spike rates $(p<0.05)$ (Fig. $4 a$ ). This was accompanied by a decrease in mean CV2 in most neurons ( $p<0.001$ for each), the most dramatic decrease observed in CINs with large CV2 values at baseline, representing phasic activity patterns (Fig. $4 c$ ). Thus, CINs tended to discharge more regularly at a higher rate after collicular disinhibition (Figs. $4 a, 5$ ), in agreement with a switch from phasic to continuous excitatory synaptic drive during ECoG activation. Linear regression analysis showed furthermore that there was a significant negative correlation between the mean spike rate and CV2 across neurons and conditions $\left(R^{2}=0.51 ; p=0.004\right.$; num- e

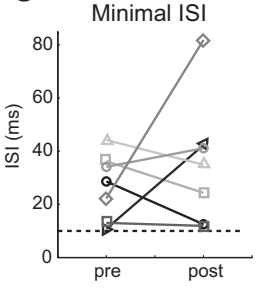

ber of observations $=14 ; F=12.41)$ (Fig. $4 d)$. This suggested a more direct link between spike rate and mean CV2. In CINs, the strong spike-AHP effectively counteracts small ISIs (Goldberg and Wilson, 2005). This was also evident in the current study in which the minimal ISI remained consistently $>10 \mathrm{~ms}$ despite the trend toward higher spike rates after BIC (Fig. 4e). Thus, increased spike regularity was a likely consequence of less patterned synaptic inputs as well as increased spike rates after BIC.

\section{Sensory-evoked spiking}

To determine whether more focused activity generated by sensory input could directly modulate spike firing of CINs, we tested the effect of a light flash presented to the rat's contralateral eye. We have recently demonstrated significant subthreshold depolarizing shifts in membrane potential in SPNs using this paradigm, but only after subcortical pathways were rendered responsive to light (Schulz et al., 2009). In agreement with this observation, no visual response was discernible in the PSTH or the membrane potential fluctuations in any CIN before BIC ejection into the SC (data not shown). However, following BIC, light stimulation induced clear changes in the spike pattern in five of seven spontaneously spiking CINs (Figs. 4-6). This was associated with the appearance of light-induced visual-evoked potentials (VEPs) in the SC (Figs. 5, 6), as reported previously (Schulz et al., 2009). The two nonresponsive CINs either showed too few trials $(n=7)$ for inclusion in the analyses or were simply devoid of any statistically significant visual-induced effects.

In responsive CINs, inspection of raster plots and PSTHs (Figs. 5, 6) showed that light stimulation regularly elicited from one or two spikes to more than five spikes, equivalent to a transient spike rate increase of $192-347 \%$ of the mean rate across neurons. Representative examples of single trials and mean responses for three CINs over this range are shown in Figures 5 and 7 , respectively. Latencies to maximal spike rate varied from 100 to $280 \mathrm{~ms}$, and the duration of the elevated spike rate varied widely from 60 to $720 \mathrm{~ms}$ between neurons. The period of increased excitation was followed by a less pronounced period of relative quiescence when the spike rate dropped below the baseline rate (Figs. 5-7). The spike rate depression started at 160-900 ms after the light flash and lasted for 380-800 ms across the five responsive neurons (Table 1). Thus, the spike rate depression in some neurons was overlapping with the spike rate elevation in other neurons. Therefore, although individual neurons exhibited a light-induced pause in tonic firing, the grand mean of the spike rate modulation across neurons preserved only the initial excitation (Fig. 7).

For statistical analysis of light-induced spike-rate modulation, taking into account the small number of light trials (range, 7-36) in individual experiments, it was necessary to convert the discrete spike times into a continuous signal. For this, we generated a 
time-resolved CV2 for each trial that measured the similarity between pairs of adjacent ISIs (see Materials and Methods). Statistical tests on this continuous signal confirmed that the spiking pattern was significantly modulated by light stimulation in five of seven neurons $(p<0.001$ for each neuron across multiple bins; Wilcoxon's signed rank test). The exact times of significant spike pattern changes varied between individual neurons, as was to be expected from the variable PSTHs (Fig. 7). In general, following the light flash, there was a broad peak in the CV2 indicating increased firing irregularity due to a continuous spike rate modulation (Fig. 7). This included the light-induced sharp increase in the spike rate and the subsequent slowing of the spike rate during the transition to a period of relative quiescence. However, in neurons that exhibited multiple spikes during the initial excitation (e.g., neuron 09410n1), the broad peak in the CV2 was delayed and preceded by a period of decreased CV2 values indicating that the high spike rate during the initial response resulted in a more regular than average firing pattern. Due to the variability in the suprathreshold response behavior between neurons, the grand mean of the time-resolved CV2 only showed a moderate tendency to increased CV2 values immediately after the light flash after BIC (Fig. 7).

\section{Light-induced membrane potential fluctuations after BIC}

To investigate subthreshold events underlying any light-evoked spike rate modulations, an average of the membrane potential was made across trials after removal of the spikes. In the five visually responsive CINs, light stimulation evoked a depolarizing PSP at a half-amplitude latency of $165 \pm 43 \mathrm{~ms}$. The PSP generally preceded the peak spike frequencies (Table 1), indicating that light-induced membrane potential fluctuations were driving the spike rate changes. The membrane potential repolarized at $618 \pm 234 \mathrm{~ms}$ entering an AHP that lasted a further $736 \pm 377 \mathrm{~ms}$ (Fig. 6). Despite the inherent interexperimental variability, the grand mean of the membrane potential modulation in Figure 7 clearly exhibited two components: an initial depolarization followed by a hyperpolarization. Thus, the dynamics of the membrane potential fluctuations were less dissimilar across experiments than the spike rate changes.

These observations indicate that the visual activation of pathways originating in the SC could powerfully modulate spiking and membrane potential fluctuations in CINs. Anatomical (Lapper and Bolam, 1992; Van der Werf et al., 2002) and physiological evidence (Matsumoto et al., 2001; Schulz et al., 2009) suggest that the thalamus may have a central role for transmitting sensory-evoked inputs to CINs in the striatum. Therefore, we next tested whether thalamo-striatal inputs could modulate tonic spiking in CINs in vitro in a similar fashion.

\section{Spike rate modulations induced by thalamic stimulation} in vitro

We used a slice preparation that allows the preferential stimulation of thalamo-striatal fibers (Smeal et al., 2007; Ding et al., 2008). Bipolar stimulation (0.5-5 mA; $0.1 \mathrm{~ms})$ through an electrode placed in the thalamus evoked PSPs of $0.6-2.8 \mathrm{mV}$ amplitude $(1.6 \pm 0.6 \mathrm{mV} ; n=15)$. The PSP amplitude was not decreased by the addition of AP5 to the bath solution, but was decreased by $92.7 \pm 1.8 \%$ in the presence of CNQX $(n=4)$ (Fig. $8 a$ ), suggesting that AMPA but not NMDA glutamate receptors contributed to the PSP in response to a single stimulus. During a stimulus train (four stimuli; $100 \mathrm{~Hz}$ ), AP5 blockade of NMDA glutamate receptors reduced the maximum amplitude of PSPs to $92.6 \pm 2.5 \%$ of controls $(6.2 \pm 4 \mathrm{mV} ; n=5 ; p<0.05)$. In spontaneously spiking CINs $(n=6 ; 0.5-1.5 \mathrm{~Hz}$ spike rate), single or a pair of stimuli had little or no effect on the postsynaptic spiking activity (Fig. 8b). However, short high-frequency (66$100 \mathrm{~Hz}$ ) bursts of four stimuli, similar to responses of parafascicular thalamic neurons to a sensory stimulus during behavior (Matsumoto et al., 2001), induced a short excitatory period followed by a pause in spike firing in the majority of spontaneously active CINs in vitro $(n=5$ of 6$)$ (Fig. $8 c$ ). The grand mean of the time-resolved CV2 exhibited a broad peak during this period. Similar to the in vivo observations, the spike rate modulations were accompanied by a statistically significant depolarizationhyperpolarization sequence in the membrane potential of five CINs 


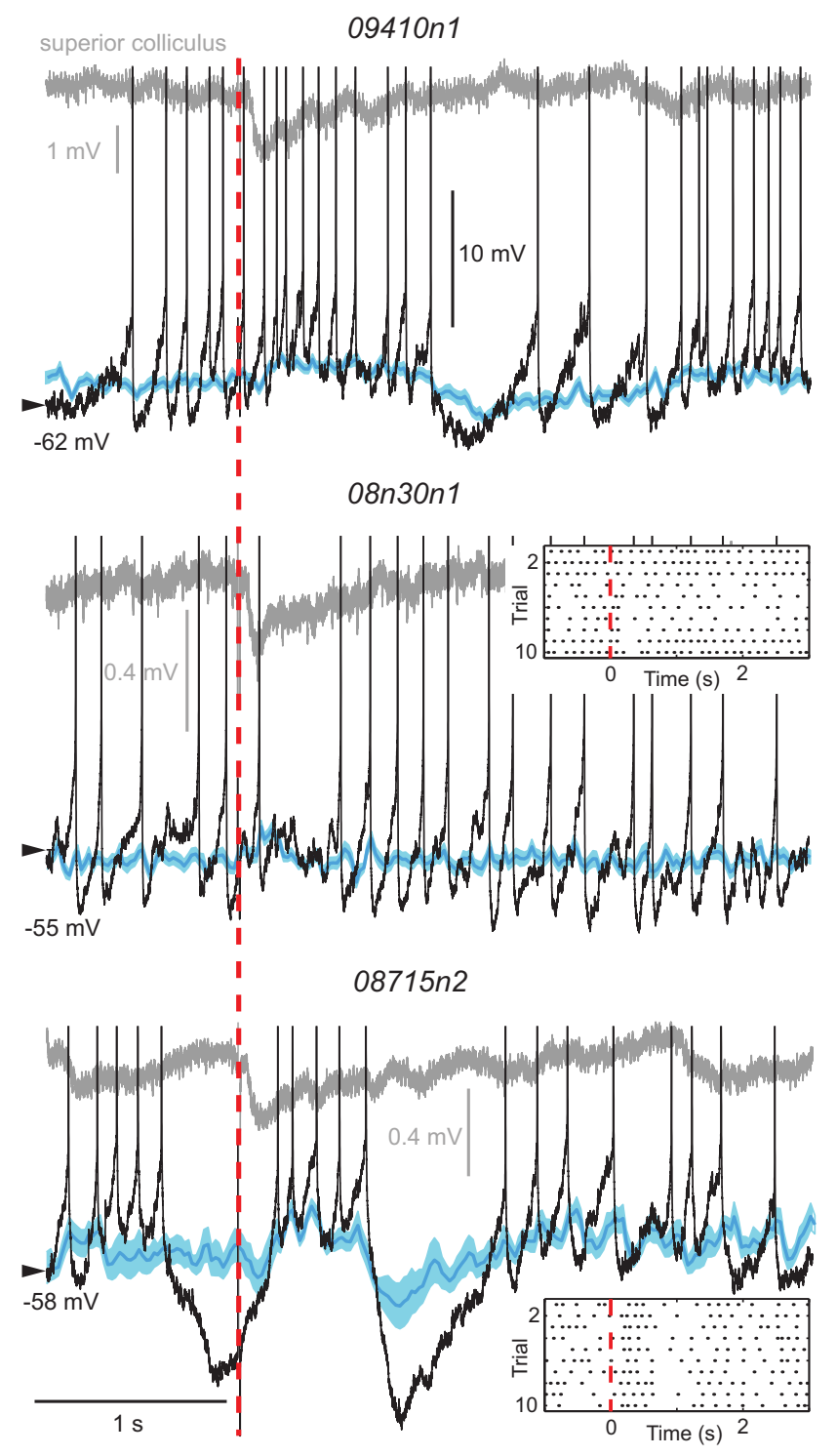

Figure 6. Representative examples of visual membrane potential responses by the same example neurons after BIC. Single light trials are aligned to the light flash onset (dashed vertical line). Scale of membrane potential fluctuations is the same for all neurons; action potentials have been truncated. The mean membrane potential trajectory across all light trials after BIC is also indicated (blue; lighter shade indicates SEM). The negative deflection in the local field potential recording from the SC marks the VEP. The insets show the spike raster plots for 10 consecutive light trials (for neuron 09410n1, see Fig. 5). Note the variability in number and timing of action potentials between neurons.

( $p<0.001$ for all neurons). This pattern persisted in the presence of the $\mathrm{GABA}_{\mathrm{A}}$ and $\mathrm{GABA}_{\mathrm{B}}$ receptor antagonists, gabazine $(10 \mu \mathrm{M})$ and (2S)-3-([(1S)-1-(3,4-dichlorophenyl)ethyl] amino-2-hydroxypropyl)(phenylmethyl)phosphinic acid (CGP55845) (1 $\mu \mathrm{M})$, respectively $(n=6)$ (Fig. $8 d$ ), and when the NMDA receptor antagonist AP5 $(50 \mu \mathrm{M})$ was added additionally $(n=2)$, indicating that it was intrinsically generated (Oswald et al., 2009).

\section{PSP-AHP coupling in vitro and in vivo}

When CINs were silent, thalamic stimulation in vitro and visual stimulation in vivo induced PSP-AHP sequences similar to those observed in spiking CINs (Fig. 9). With the influence of large spike-associated membrane potential fluctuations absent, it was possible to examine in detail the relationship between lightevoked synaptic inputs and the subsequent AHP. In vitro, the
AHP area was directly proportional to the PSP area of thalamic responses in the presence and absence of gabazine $(n=5)$ (Fig. $9 a, b)$. This is consistent with the hypothesis that evoked excitatory synaptic inputs and an intrinsically generated AHP together underlie the spike rate modulation. In vivo, a similar PSP-AHP relationship could be observed in two CINs during light trials where spike discharge was absent (Fig. 9). In one experiment with a sufficient number of subthreshold light trials, the PSP area was significantly correlated with AHP area (Fig. 9c) and PSP amplitude with the duration of the AHP $\left(R^{2}=0.49 ; p=0.025\right)$. In addition, for the group of spontaneously active CINs there was a significant correlation between AHP area and PSP area for the mean visual responses $\left(R^{2}=0.82\right)$ (Fig. $\left.9 e\right)$. These observations further support the hypothesis of an intrinsically generated AHP as a common mechanism for the transient spike rate depression in CINs.

The SC was most likely the indirect source of visual-evoked inputs to striatal CINs in our in vivo experiments (Schulz et al., 2009). In agreement with this, the amplitude and area of the PSP were positively correlated with the VEP amplitude recorded in the SC in recordings of one of the two silent CINs (Fig. $9 d$ ). Thus, visual response parameters in CINs appeared to depend on response strength in the SC in a similar fashion as previously observed on a larger sample of striatal SPNs (Schulz et al., 2009). Together, the present results therefore showed for the first time that visual events detected by the SC and relayed by the thalamus powerfully modulate spiking in CINs in the striatum. These excitatory inputs directly evoked a membrane depolarization and spike response followed by an intrinsically generated AHP underlying a pause in tonic firing (Fig. 10).

\section{Discussion}

Sensory responses in TANs during behavioral conditioning have been extensively studied in the hope to gain insights into striatal mechanisms of reward-based learning. Yet the anatomical pathways and cellular mechanisms involved remain poorly understood. Here, using rarely obtained intracellular recordings from these neurons in vivo (Wilson et al., 1990; Reynolds and Wickens, 2004; Reynolds et al., 2004), we show for the first time that visual stimuli induce a PSP-AHP sequence in CINs that underlies an excitation-pause sequence in spiking activity. These responses depended on the disinhibition of the SC, strongly implicating tecto-striatal pathways in the regulation of CIN and TAN spiking activity.

\section{Regulation of the spontaneous activity pattern}

The CINs recorded in the current study exhibited a broad spectrum of spontaneous activity patterns, including tonic regular firing, phasic activity patterns, and even long periods of quiescence. This diversity was surprising given that urethane anesthesia induced a slow-wave EEG pattern in all experiments, associated with highly stereotypical membrane potential fluctuations and firing patterns in SPNs (Reynolds and Wickens, 2004; Schulz et al., 2009). For CINs, however, a similarly broad range of activity patterns has been reported in brain slices even after the blockade of synaptic inputs (Bennett and Wilson, 1999). Results from the present study confirm this heterogeneity in vivo and suggest that, in CINs, the spike output pattern is largely determined by intrinsic properties. In addition, the present study demonstrates that the more phasic activity patterns tend to be entrained to the slow-wave activity in afferent networks. Both the degree of spike-ECoG coupling and the mean CV2 value appeared to cover a continuous range of values across the CIN population. Together, the present results therefore indicate that, in vivo, phasic bursting and regular tonic spiking are at the 
extremes of a continuous distribution, with most CINs falling in between these extremes.

After BIC ejection into the SC, the activity pattern was shifted toward faster and more regular spiking. Two mechanisms likely contributed to the changes reported here. First, pharmacological or electrical stimulation of the SC induces an altered neuromodulator milieu in the forebrain via brainstem activation (Dringenberg et al., 2003) and consequently high-frequency, low-amplitude activation of the EEG (Dringenberg et al., 2003; Schulz et al., 2009). Subsequently, glutamatergic inputs tonically depolarized CINs and ceased to support more phasic spiking patterns. Second, coincident increases in both serotonin and DA levels in the striatum (Dringenberg et al., 2003; Dommett et al., 2005) probably directly contributed to the increased spike rate by increasing excitability (Aosaki et al., 1998; Blomeley and Bracci, 2005; Deng et al., 2007). Limited by the strong CIN-inherent spike-AHP (Goldberg and Wilson, 2005), increased spiking regularity was a consequence of increased and unpatterned excitation. In agreement with this interpretation, direct stimulation of the substantia nigra pars compacta initially induces fast and regular spiking, later followed by rhythmic bursting (Reynolds et al., 2004). These observations clearly demonstrate that the spiking pattern of CINs in vivo is dynamically regulated by an interplay between intrinsic and extrinsic factors.

\section{Phasic activation by visual-evoked inputs}

Disinhibition of the SC was essential to enable visual activation of CINs. After BIC, visual stimulation induced a depolarization at latencies $(\sim 165 \mathrm{~ms})$ comparable with the large PSPs observed previously in SPNs ( $\sim 120 \mathrm{~ms})$ (Schulz et al., 2009). In contrast to SPNs in which no change in spike output was apparent, visual inputs to CINs regularly induced a transient spike rate increase, typically followed by a pause in firing. The thalamus provides the most likely anatomical route for relaying visual-evoked activity from the SC to the striatum (Van der Werf et al., 2002). We have confirmed that the parafascicular nucleus in particular is important for short-latency visual responses in SPNs (Schulz et al., 2009). Similarly, pharmacological inhibition of the parafascicular nucleus homolog in monkeys suppresses responses in TANs to behaviorally significant stimuli (Matsumoto et al., 2001). Hence, we proceeded to test directly the effectiveness of thalamo-striatal inputs in regulating spike output of CINs.

Repetitive stimulation of thalamo-striatal afferents in vitro evoked a stereotypic spike-pause response resulting from the underlying PSP-AHP sequence. Intrinsic AHPs in response to sustained depolarization are a characteristic property of CINs (Goldberg and Wilson, 2005; Deng et al., 2007) (Fig. 1) and are likely to be elicited by prolonged synaptic inputs such as that engaged by visual stimulation (Schulz et al., 2009). Upon the relaxation of depolarizing synaptic currents, the membrane potential is dominated by the slow AHP-type $\mathrm{Ca}^{2+}$-activated $\mathrm{K}^{+}$ currents $\left(I_{\text {sAHP }}\right)$ (Goldberg and Wilson, 2005) and will hyperpo-

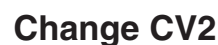

Membrane potential
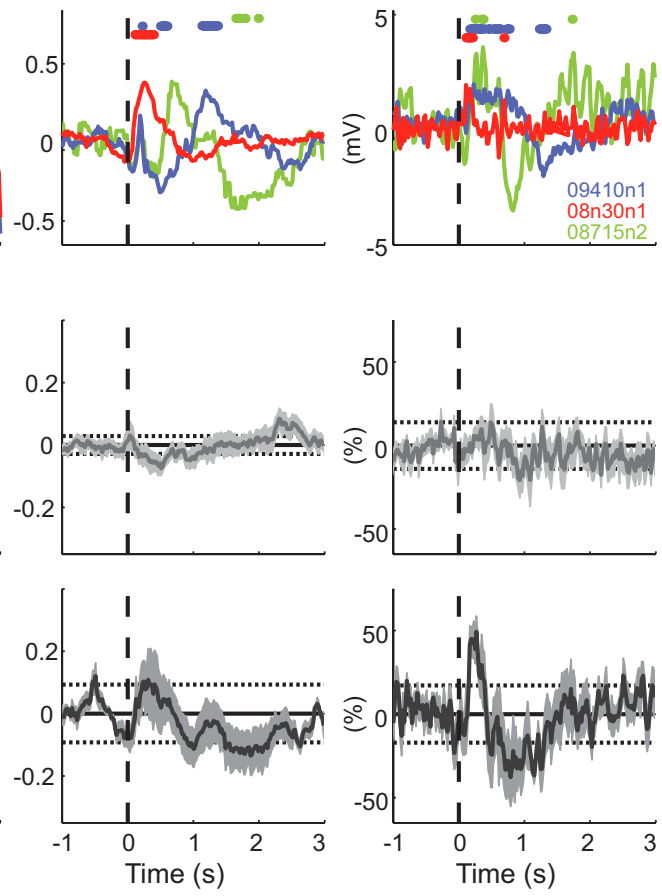

Figure 7. Mean modulation of spike rate, time-resolved CV2, and membrane potential induced by light flash stimulation before line are indicated by colored dots ( $p<0.001$. Wilcoxon's signed rank test). Note that the time course of light-evoked modulations varied largely between neurons. The grand means (7 neurons) for responses before and after BIC ejection are shown in the second and third rows, respectively. The lighter shades indicate the SEM. The dotted lines indicate 2 SD from prelight mean.

larize. Small, brief subthreshold depolarizations in response to cortical synaptic inputs are often followed by AHPs (Reynolds et al., 2004), where the principal mechanism is the transient deactivation of the $I_{\mathrm{H}}$ (Oswald et al., 2009). Thus, CINs appear to possess multiple intrinsic mechanisms to generate membrane potential hyperpolarizations following excitation that function over a broad range of magnitudes and timescales.

An unexpected observation in the present study was the significant variability of responses between individual CINs in vivo compared with our in vitro observations. Potentially the most important difference between the two preparations was the relative degree of synaptic input activation. We used short well defined bursts of highfrequency stimuli in vitro, similar to spike patterns observed in awake animals (Matsumoto et al., 2001). In contrast, visual stimuli during collicular disinhibition in vivo resulted in longer-lasting activation of afferent neurons, as evident from recordings in the SC (Coizet et al., 2003; Dommett et al., 2005) and visual response characteristics in SPNs (Schulz et al., 2009). Similar variability in pauses in CIN activity has been reported recently in response to prolonged highfrequency stimulation of the thalamus in vitro (Ding et al., 2010). Thus, differences in our in vivo visual responses following BIC compared with those typically reported in TANs likely reflect more widespread and prolonged recruitment of the inputs normally activated by behaviorally significant visual stimuli.

It is noteworthy that the collicular inputs driving visual responsiveness in CINs interact with a striatal circuit that affects voluntary control of saccades (SC-thalamus-striatum-basal ganglia output nuclei-SC). While interneurons will not be able to control saccades directly, they could influence voluntary control by modulating cortical input to spiny neurons during the pause (Ding et al., 

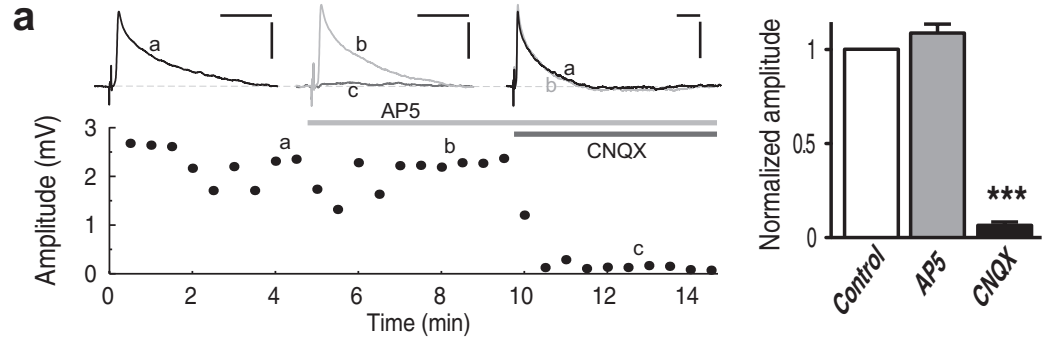

b
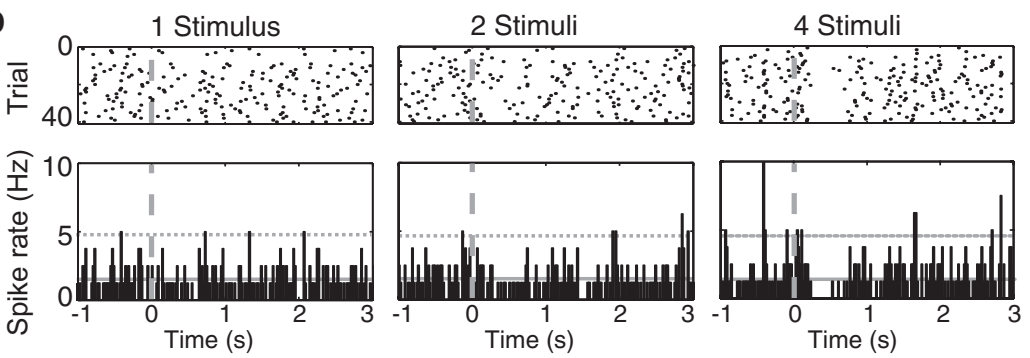

C
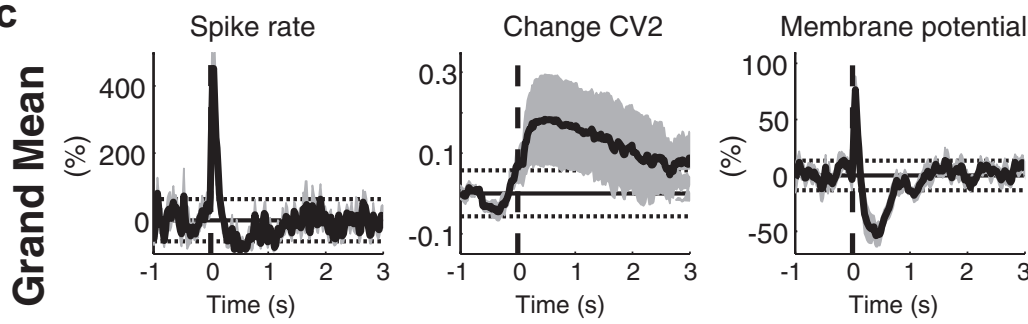

d
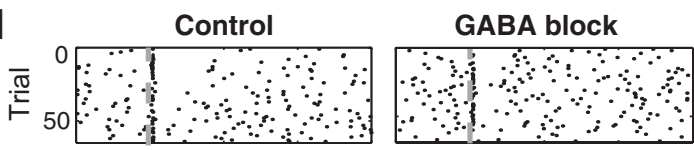

GABA block + AP5
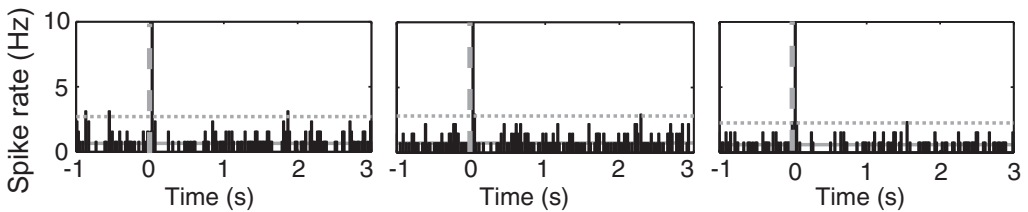

Figure 8. Stimulation of thalamo-striatal afferents evokes excitatory PSPs and spike-pause responses in CINs in vitro.a, Characteristics of thalamo-striatal PSPs. Singlestimulation within the thalamus or the internal capsule evoked depolarizing PSPs in CINs that were resistant to $50 \mu \mathrm{m}$ APV but were blocked by $10 \mu \mathrm{m}$ CNQX. Single neuron example of PSP amplitudes (averages of 6 traces recorded at a $5 \mathrm{~ms}$ interval), illustrating the effect of application of AP5 and CNQX. Representative traces of 1 min averages at the time points a, $b$, and c are indicated. Calibration: $1.5 \mathrm{mV}, 50 \mathrm{~ms}$. Right, Average responses following 3-5 min of drug application (normalized to the mean control PSP amplitude) showed that the PSP was blocked by $93 \%$ by CNQX ( $n=4 ;{ }^{* * *} p<0.001$, one-way ANOVA and Dunnett's post hoc analysis). Error bars indicate SEM. $\boldsymbol{b}$, Raster plots and PSTHs from an example experiment show that PSPs evoked by repetitive stimulation $(66-100 \mathrm{~Hz})$, but not by one to two stimuli were sufficient to induce a spike-pause response in CINs. c, The grand mean (6 neurons) of spike-rate modulation, time-resolved CV2, and membrane potential induced by four thalamo-striatal stimuli. Note the consistent induction of a spike-pause response and underlying PSP-AHP sequence. $d$, Raster plots and PSTHs from an example experiment show that spike-pause responses to thalamo-striatal PSPs persisted in the presence of the GABA antagonists gabazine and CGP55845, and with the further addition of the NMDA receptor antagonist AP5.

2010). In fact, CINs may deliver a sensory bottom-up signal that is active during periods that require increased top-down control. However, it is not known how CIN pause responses interact with thalamic inputs to spiny neurons, which are activated in parallel. In our preparation, thalamic inputs to SPNs do not favor increased spiking in response to visual input (Schulz et al., 2009), supporting the hypothesis presented by Ding et al. (2010).

\section{Interaction between cholinergic and dopaminergic neuron responses}

In TANs recorded in behaving monkeys, the continued expression of established pause responses is dependent on the presence of intact DA transmission (Aosaki et al., 1994). In our study, DA neurons were probably simultaneously driven by the light flash, since BIC ejection into the SC induces visual responsiveness in DA neurons (Fig. 10) (Coizet et al., 2003; Dommett et al., 2005). However, a number of pieces of indirect evidence suggest that visual-evoked phasic DA release was not directly necessary for the expression of visual responsiveness in CINs. First, the net effect of DA on the CIN membrane is depolarizing rather than hyperpolarizing (Aosaki et al., 1998; Deng et al., 2007). Second, tonic activation of DA receptors by systemic application of apomorphine is sufficient to reverse loss of TAN responses in the DA-depleted striatum (Aosaki et al., 1994). Third, TANs do not show the same reward responses as DA neurons (Morris et al., 2004; Joshua et al., 2008), suggesting that activation of DA neurons is not essential for the pause. Fourth, thalamicinduced pauses in CIN firing are attenuated but not abolished by blockade of the local nicotinic receptor mechanism that likely drives dopamine release in a deafferented slice preparation (Ding et al., 2010). Finally, in behaving animals, thalamic neurons respond strongly to sensory stimuli during conditions when neither TANs nor DA neurons respond (Matsumoto et al., 2001), making it likely that the DA projection contributes independently from thalamic inputs to enable TAN responses. Possible indirect roles for the DA input might include induction of short-term (Salgado et al., 2005) or longterm synaptic plasticity (Reynolds et al., 2004) that boosts the impact of depolarizing synaptic inputs to CINs, or DAdependent modulation of dendritic integration in CINs through downregulation of $I_{\mathrm{H}}$ (Nolan et al., 2004; Campanac et al., 2008). Although it is clear that DA plays some role in the expression of pause responses of TANs, the exact mechanisms need to be elucidated through further research.

In awake monkeys, TANs and DA neurons exhibit coincident responses to visual stimuli (Morris et al., 2004; Joshua et al., 2008). The present results suggest a mechanism: that DA neurons and CINs may both be activated via the SC. While direct collicular inputs cause burst firing in DA neurons (Dommett et al., 2005; Joshua et al., 2008), the same collicular neurons possess axon collaterals that project to the parafascicular nucleus of the thalamus (Coizet et al., 2007). We think that the divergence of salient signals in the SC into tecto-nigro-striatal and tecto-thalamo-striatal pathways could be an efficient anatomical implementation to ensure that the DA burst coincides with a pause in ACh tone, which has been suggested to boost DA release and to facilitate synaptic processing in SPNs (Morris et al., 2004; Cragg, 2006; Wang et al., 2006). 


\section{IN VITRO}

a

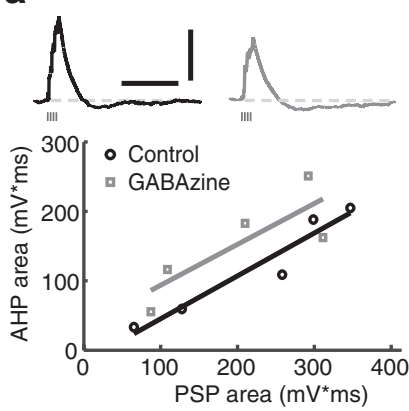

b

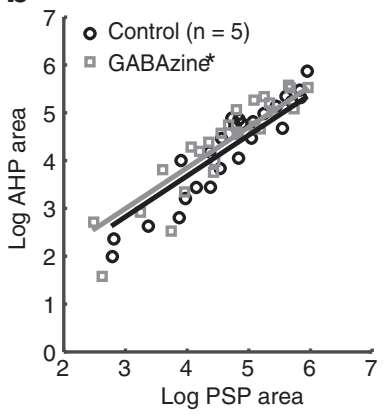

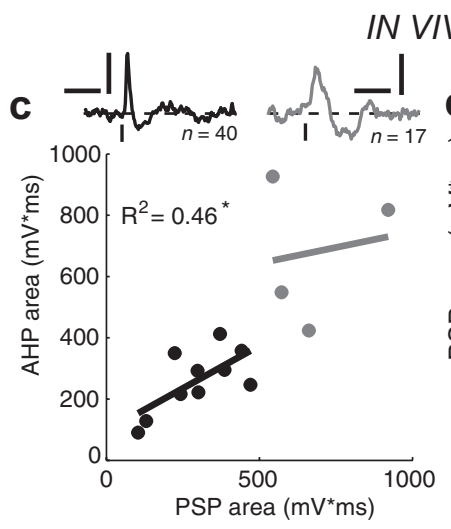

e

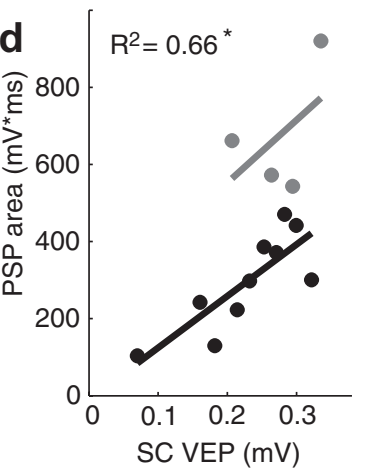

$\mathrm{SC}$ VEP $(\mathrm{mV})$

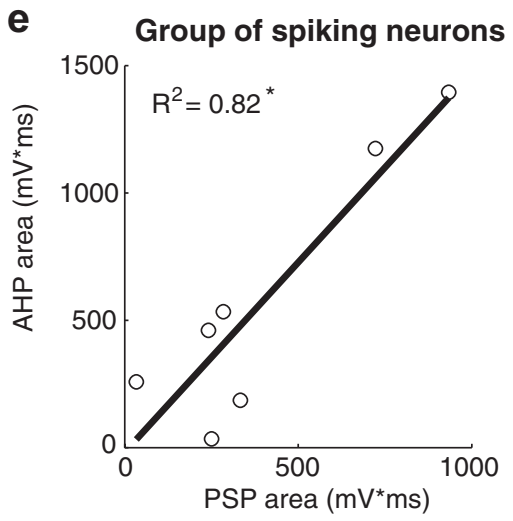

Figure 9. PSP and AHP area are correlated in in vitro and in vivo experiments. $\boldsymbol{a}$, Representative example of a scatter plot of AHP versus PSP area measurements in the absence and presence of GABA antagonists gabazine and CGP55845 in subthreshold trials in vitro. Data points are mean areas of episodes grouped by the number of stimuli in a train. Average traces for each condition are shown above for four stimuli. Calibration: $3 \mathrm{mV}, 200 \mathrm{~ms}$. $\boldsymbol{b}$, Group analysis of in vitro experiments with subthreshold PSPs show that the correlation between PSP and AHP persisted in the presence of GABA antagonists that appeared to enhance the AHP (mixed-model regression, $\left.{ }^{*} p<0.05\right)$. c, Scatter plot of AHP versus PSP parameters of subthreshold visual responses from trials without AP discharge in two in vivo experiments. The filled circles represent measurements on averages of four episodes. The insets show the mean response. Calibration: $2 \mathrm{mV}, 1 \mathrm{~s}$. The time of the light flash and number included individual trials are indicated. The $R^{2}$ value indicates the correlation in one experiment $\left({ }^{*} p<0.05\right) . \boldsymbol{d}$, PSP area was positively correlated to collicular response strength in the subthreshold responses of one neuron. $\boldsymbol{e}$, The circles represent the mean visual responses of the group of spontaneously active neurons in vivo. There was a significant correlation between AHP area and PSP area.

\section{References}

Aosaki T, Graybiel AM, Kimura M (1994) Effect of the nigrostriatal dopamine system on acquired neural responses in the striatum of behaving monkeys. Science 265:412-415.

Aosaki T, Kiuchi K, Kawaguchi Y (1998) Dopamine $D_{1}$-like receptor activation excites rat striatal large aspiny neurons in vitro. J Neurosci 18: $5180-5190$.

\section{Visual activation of tecto-striatal inputs onto CINs}

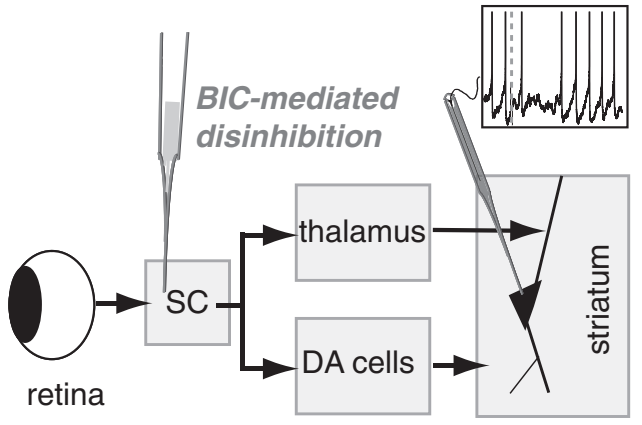

Figure 10. A model of the pathways involved in mediating visual responses in cholinergic interneurons. Tecto-thalamic inputs directly drive spike-pause responses in CINs when they are sufficiently strong (e.g., after BIC ejection into the $\mathrm{SC}$ in the current study). Simultaneous responses in CINs and DA neurons can interact to modulate information processing in the striatum.

Bennett BD, Wilson CJ (1999) Spontaneous activity of neostriatal cholinergic interneurons in vitro. J Neurosci 19:5586-5596.

Bennett BD, Callaway JC, Wilson CJ (2000) Intrinsic membrane properties underlying spontaneous tonic firing in neostriatal cholinergic interneurons. J Neurosci 20:8493-8503.

Blomeley C, Bracci E (2005) Excitatory effects of serotonin on rat striatal cholinergic interneurones. J Physiol 569:715-721.

Campanac E, Daoudal G, Ankri N, Debanne D (2008) Downregulation of dendritic $I_{\mathrm{h}}$ in CAl pyramidal neurons after LTP. J Neurosci 28:8635-8643.

Clement EA, Richard A, Thwaites M, Ailon J, Peters S, Dickson CT (2008) Cyclic and sleep-like spontaneous alternations of brain state under urethane anaesthesia. PLoS ONE 3:e2004.

Coizet V, Comoli E, Westby GW, Redgrave P (2003) Phasic activation of substantia nigra and the ventral tegmental area by chemical stimulation of the superior colliculus: an electrophysiological investigation in the rat. Eur J Neurosci 17:28-40.

Coizet V, Overton PG, Redgrave P (2007) Collateralization of the tectonigral projection with other major output pathways of superior colliculus in the rat. J Comp Neurol 500:1034-1049.

Cragg SJ (2006) Meaningful silences: how dopamine listens to the ACh pause. Trends Neurosci 29:125-131.

Davies RM, Gerstein GL, Baker SN (2006) Measurement of time-dependent changes in the irregularity of neural spiking. J Neurophysiol 96:906-918.

DeBoer P, Abercrombie ED (1996) Physiological release of striatal acetylcholine in vivo: modulation by D1 and D2 dopamine receptor subtypes. J Pharmacol Exp Ther 277:775-783.

Deng P, Zhang Y, Xu ZC (2007) Involvement of $I_{\mathrm{h}}$ in dopamine modulation of tonic firing in striatal cholinergic interneurons. J Neurosci 27:3148-3156.

Ding J, Peterson JD, Surmeier DJ (2008) Corticostriatal and thalamostriatal synapses have distinctive properties. J Neurosci 28:6483-6492.

Ding JB, Guzman JN, Peterson JD, Goldberg JA, Surmeier DJ (2010) Thalamic gating of corticostriatal signaling by cholinergic interneurons. Neuron 67:294-307.

Dommett E, Coizet V, Blaha CD, Martindale J, Lefebvre V, Walton N, Mayhew JE, Overton PG, Redgrave P (2005) How visual stimuli activate dopaminergic neurons at short latency. Science 307:1476-1479.

Dringenberg HC, Vanderwolf CH, Noseworthy PA (2003) Superior colliculus stimulation enhances neocortical serotonin release and electrocorticographic activation in the urethane-anesthetized rat. Brain Res 964:31-41.

Fries P, Reynolds JH, Rorie AE, Desimone R (2001) Modulation of oscillatory neuronal synchronization by selective visual attention. Science 291:1560-1563.

Goldberg JA, Wilson CJ (2005) Control of spontaneous firing patterns by the selective coupling of calcium currents to calcium-activated potassium currents in striatal cholinergic interneurons. J Neurosci 25:10230-10238.

Grillner S, Hellgren J, Ménard A, Saitoh K, Wikström MA (2005) Mechanisms for selection of basic motor programs-roles for the striatum and pallidum. Trends Neurosci 28:364-370.

Holt GR, Softky WR, Koch C, Douglas RJ (1996) Comparison of discharge 
Table 1. Mean visual response parameters in visually responsive spontaneously spiking CINs after BIC

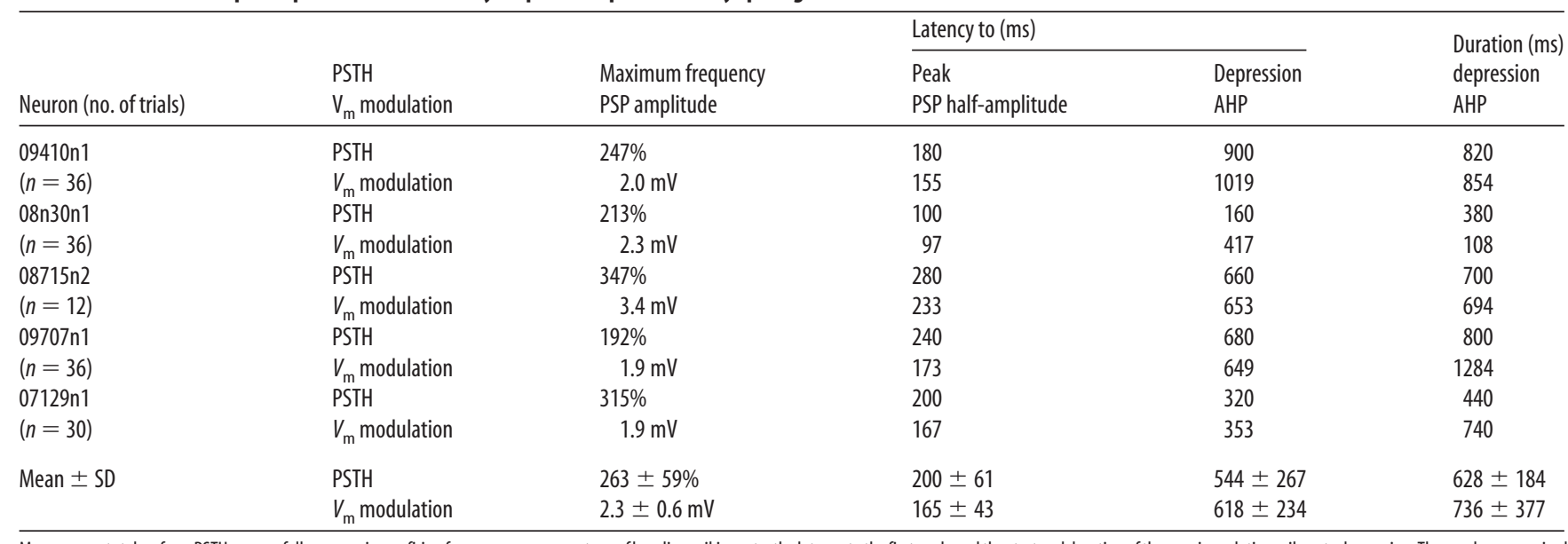

Measurements taken from PSTH were as follows: maximum firing frequency as a percentage of baseline spiking rate, the latency to the first peak, and the start and duration of the ensuing relative spike rate depression. These values are paired with the amplitude of the light-induced PSP, the latency to PSP half-amplitude, and the start and duration of the subsequent AHP for comparison.

variability in vitro and in vivo in cat visual cortex neurons. J Neurophysiol 75:1806-1814.

Horikawa K, Armstrong WE (1988) A versatile means of intracellular labeling: injection of biocytin and its detection with avidin conjugates. J Neurosci Methods 25:1-11.

Joshua M, Adler A, Mitelman R, Vaadia E, Bergman H (2008) Midbrain dopaminergic neurons and striatal cholinergic interneurons encode the difference between reward and aversive events at different epochs of probabilistic classical conditioning trials. J Neurosci 28:11673-11684.

Kawaguchi Y, Wilson CJ, Augood SJ, Emson PC (1995) Striatal interneurones: chemical, physiological and morphological characterization. Trends Neurosci $18: 527-535$.

Kimura M (1990) Behaviorally contingent property of movement-related activity of the primate putamen. J Neurophysiol 63:1277-1296.

Kudernatsch M, Sutor B (1994) Cholinergic modulation of dopamine overflow in the rat neostriatum - a fast cyclic voltammetric study in vitro. Neurosci Lett 181:107-112.

Lapper SR, Bolam JP (1992) Input from the frontal cortex and the parafascicular nucleus to cholinergic interneurons in the dorsal striatum of the rat. Neuroscience 51:533-545.

Matsumoto N, Minamimoto T, Graybiel AM, Kimura M (2001) Neurons in the thalamic CM-Pf complex supply striatal neurons with information about behaviorally significant sensory events. J Neurophysiol 85:960-976.

Maurice N, Mercer J, Chan CS, Hernandez-Lopez S, Held J, Tkatch T, Surmeier DJ (2004) $\mathrm{D}_{2}$ dopamine receptor-mediated modulation of voltage-dependent $\mathrm{Na}^{+}$channels reduces autonomous activity in striatal cholinergic interneurons. J Neurosci 24:10289-10301.

Morris G, Arkadir D, Nevet A, Vaadia E, Bergman H (2004) Coincident but distinct messages of midbrain dopamine and striatal tonically active neurons. Neuron 43:133-143.

Nolan MF, Malleret G, Dudman JT, Buhl DL, Santoro B, Gibbs E, Vronskaya S, Buzsáki G, Siegelbaum SA, Kandel ER, Morozov A (2004) A behavioral role for dendritic integration: HCN1 channels constrain spatial inputs to distal dendrites memory and plasticity at of CA1 pyramidal neurons. Cell 119:719-732.

Oorschot DE (1996) Total number of neurons in the neostriatal, pallidal, subthalamic, and substantia nigral nuclei of the rat basal ganglia: a stereological study using the Cavalieri and optical disector methods. J Comp Neurol 366:580-599.

Oswald MJ, Oorschot DE, Schulz JM, Lipski J, Reynolds JN (2009) $I_{\mathrm{H}}$ current generates the afterhyperpolarisation following activation of subthreshold cortical synaptic inputs to striatal cholinergic interneurons. J Physiol 587:5879-5897.

Pakhotin P, Bracci E (2007) Cholinergic interneurons control the excitatory input to the striatum. J Neurosci 27:391-400.

Pisani A, Bernardi G, Ding J, Surmeier DJ (2007) Re-emergence of striatal cholinergic interneurons in movement disorders. Trends Neurosci 30:545-553.

Reynolds JN, Wickens JR (2004) The corticostriatal input to giant aspiny interneurons in the rat: a candidate pathway for synchronising the response to reward-related cues. Brain Res 1011:115-128.
Reynolds JN, Hyland BI, Wickens JR (2004) Modulation of an afterhyperpolarization by the substantia nigra induces pauses in the tonic firing of striatal cholinergic interneurons. J Neurosci 24:9870-9877.

Reynolds JNJ, Williams JM, Schulz JM (2009) Induction of pause responses in striatal cholinergic interneurons by sensory stimuli: an in vivo intracellular study. Soc Neurosci Abstr 35:845.1.

Salgado H, Tecuapetla F, Perez-Rosello T, Perez-Burgos A, Perez-Garci E, Galarraga E, Bargas J (2005) A reconfiguration of $\mathrm{Ca}_{\mathrm{V}} 2 \mathrm{Ca}^{2+}$ channel current and its dopaminergic D2 modulation in developing neostriatal neurons. J Neurophysiol 94:3771-3787.

Schulz JM, Redgrave P, Mehring C, Aertsen A, Clements KM, Wickens JR, Reynolds JN (2009) Short-latency activation of striatal spiny neurons via subcortical visual pathways. J Neurosci 29:6336-6347.

Schulz JM, Redgrave P, Reynolds JN (2010) Cortico-striatal spike-timing dependent plasticity after activation of subcortical pathways. Front Synaptic Neurosci 2:23.

Shen W, Tian X, Day M, Ulrich S, Tkatch T, Nathanson NM, Surmeier DJ (2007) Cholinergic modulation of Kir2 channels selectively elevates dendritic excitability in striatopallidal neurons. Nat Neurosci 10:1458-1466.

Shin SL, Hoebeek FE, Schonewille M, De Zeeuw CI, Aertsen A, De Schutter E (2007) Regular patterns in cerebellar Purkinje cell simple spike trains. PLoS One 2:e485.

Smeal RM, Gaspar RC, Keefe KA, Wilcox KS (2007) A rat brain slice preparation for characterizing both thalamostriatal and corticostriatal afferents. J Neurosci Methods 159:224-235.

Sullivan MA, Chen H, Morikawa H (2008) Recurrent inhibitory network among striatal cholinergic interneurons. J Neurosci 28:8682-8690.

Van der Werf YD, Witter MP, Groenewegen HJ (2002) The intralaminar and midline nuclei of the thalamus. Anatomical and functional evidence for participation in processes of arousal and awareness. Brain Res Rev 39:107-140.

Wang Z, Kai L, Day M, Ronesi J, Yin HH, Ding J, Tkatch T, Lovinger DM, Surmeier DJ (2006) Dopaminergic control of corticostriatal long-term synaptic depression in medium spiny neurons is mediated by cholinergic interneurons. Neuron 50:443-452.

Wilson CJ (2005) The mechanism of intrinsic amplification of hyperpolarizations and spontaneous bursting in striatal cholinergic interneurons. Neuron 45:575-585.

Wilson CJ, Goldberg JA (2006) Origin of the slow afterhyperpolarization and slow rhythmic bursting in striatal cholinergic interneurons. J Neurophysiol 95:196-204.

Wilson CJ, Chang HT, Kitai ST (1990) Firing patterns and synaptic potentials of identified giant aspiny interneurons in the rat neostriatum. J Neurosci 10:508-519.

Yan Z, Song WJ, Surmeier J (1997) D2 dopamine receptors reduce N-type $\mathrm{Ca}^{2+}$ currents in rat neostriatal cholinergic interneurons through a membrane-delimited, protein-kinase-C-insensitive pathway. J Neurophysiol 77:1003-1015. 